\title{
Modeling Secondary Electron Imaging at Atomic Resolution Using a Focused Coherent Electron Probe
}

\author{
L. J. Allen ${ }^{1}$, H. G. Brown ${ }^{1}$, A. J. D’Alfonso ${ }^{1}$, J. Ciston $^{2}$, Y. Lin ${ }^{3}$ and L. D. Marks ${ }^{3}$ \\ ${ }^{1}$ School of Physics, University of Melbourne, Parkville, Victoria 3010, Australia \\ ${ }^{2}$ National Center for Electron Microscopy, Lawrence Berkeley National Laboratory, Berkeley, \\ California 94720, USA \\ ${ }^{3}$ Department of Materials Science and Engineering, Northwestern University, 2220 Campus Drive, \\ Evanston, Illinois 60208, USA
}

In scanning transmission electron microscopy (STEM), the techniques of annular dark field imaging, electron energy loss spectroscopy, and, more recently, energy dispersive x-ray imaging all provide atomic resolution imaging. In the area of STEM using secondary electrons this has been more elusive, the first atomic resolution images only being demonstrated as recently as 2009 [1].

Consider the entrance surface of a specimen irradiated by primary fast electrons with energy of the order of $100 \mathrm{keV}$. Electrons are observed to be emitted from the entrance surface. Those with energies less than around $50 \mathrm{eV}$ are the secondary electrons to which we refer [2]. Electrons with energies between around $50 \mathrm{eV}$ and the energy of the incident electrons are mainly Auger electrons and thermally (Rutherford) backscattered electrons [2]. In the experiment of Zhu et al. [1] 85-90\% of the electrons detected in the backwards direction had a kinetic energy between 0 and $50 \mathrm{eV}$, with most having an energy less than $10 \mathrm{eV}$. We assume that these low-energy electrons are predominantly the product of ionization events. For an atomically sized probe, localized (core-shell) ionization provides high resolution (bulk) atomic column positions.

We have developed a quantum mechanical model to understand such secondary electron images [3]. A key component is the angular probability distribution of the electrons ejected in the ionization events. The angular distributions are most strongly influenced by the bound state angular momentum quantum number, as can be seen in Fig. 1. Electrons ejected with low energy have a short mean free path for inelastic scattering. The probability that an ejected electron is absorbed in the specimen rather than being detected is a function not only of its energy and the depth in the specimen at which ionization occurs, but also of the direction in which it is emitted, since this influences the path length to the surface. The model described allowed us to match in detail features in the experimental data of Zhu et al. [1], as can be seen in Fig. 2. A modest number of atomic orbitals were required to understand the image contrast. Electrons with too low an ionization threshold, often part of the valence band, do not contribute atomic scale information while those with a large ionization threshold were only responsible for a small fraction of the signal.

The fact that low energy secondary electrons emitted more than a few atomic layers into the specimen are absorbed and not detected suggests that secondary electron imaging should be a good technique for exploring surfaces [4]. In Fig. 3 we show a preliminary simulation of secondary electron data obtained for a $\mathrm{c}(6 \times 2)(\mathrm{c} 2 \mathrm{~mm}$ symmetry) unit cell surface reconstruction on $<100>$ strontium titanate. Here both core and semi-core shells contribute. The proposed structure was refined using density functional theory and is consistent with scanning tunneling microscopy, Auger, surface x-ray and transmission electron microscopy diffraction and high-resolution imaging data. 


\section{References:}

[1] Y Zhu et al., Nat. Mater. 8 (2009) p. 808.

[2] H Seiler, J. Appl. Phys. 54 (1983) p. R1.

[3] HG Brown et al., Phys. Rev. B 87 (2013) 054102.

[4] A Howie, J. Microsc. 180 (1995) p.192.

[5] This research was supported under Australian Research Council's Discovery Projects funding scheme (Project No. DP1 10102228).

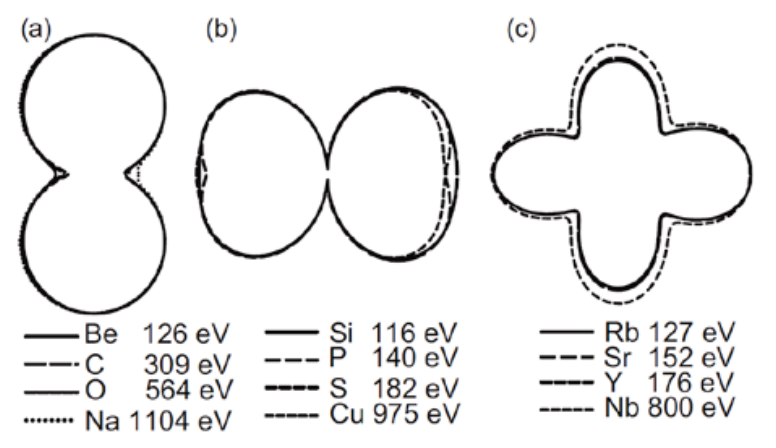

Figure 1. Normalized doubledifferential cross sections (angular distributions) for the ionization involving (a) $1 \mathrm{~s}$, (b) $2 \mathrm{p}$, and (c) $3 \mathrm{~d}$ electrons for different atoms at $10 \mathrm{eV}$ above the respective ionization thresholds. The primary fast electron (200 keV, plane wave) is incident rom the left. The results include integration up over all possible scattering directions of the scattered fast (energyloss) electron.

Figure 2. Images of a YBCO

specimen. The $200-\mathrm{keV}$ probe had a convergence semi-angle of $28 \mathrm{mrad}$. (a) The experimental image taken by Zhu et al. [1] and (b) the simulated image, taking into account the angular dependence of the ejected secondary electrons and their absorption in the specimen. Simulations assumed a specimen thickness of $50 \AA$ and a

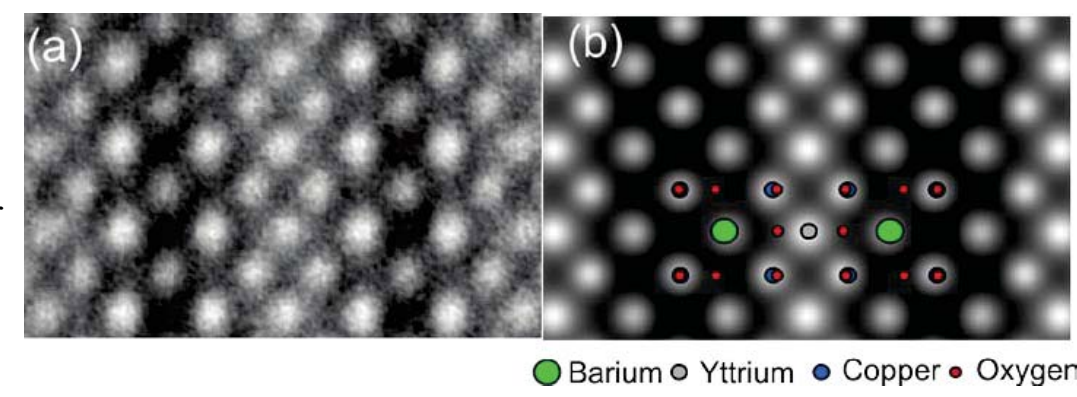
finite source size of FWHM $1 \AA$.

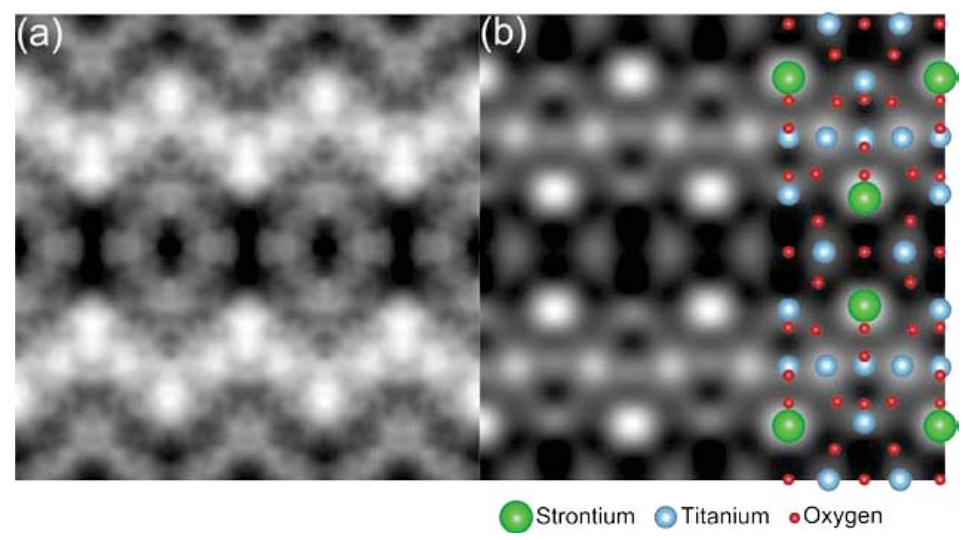

Figure 3. (a) Bulk subtracted secondary electron image of a $6 \times 2$ unit cell surface reconstruction on a $<100>$ strontium titanate substrate. The $200-\mathrm{keV}$ probe had a convergence semi-angle of 25 mrad. (b) Simulation of the result in (b) using the (projected) surface reconstruction indicated. 